\title{
Anti-ovarian tumor response of donor peripheral blood mononuclear cells is due to infiltrating cytotoxic NK cells
}

\author{
Veethika Pandey ${ }^{1}$, Jeremiah L. Oyer ${ }^{1}$, Robert Y. Igarashi ${ }^{1}$, Sarah B. Gitto ${ }^{1}$, Alicja J. \\ Copik $^{1}$, Deborah A. Altomare ${ }^{1}$ \\ ${ }^{1}$ Burnett School of Biomedical Sciences, College of Medicine, University of Central Florida, Orlando, FL, USA \\ Correspondence to: Deborah A. Altomare, e-mail: Deborah.Altomare@ucf.edu \\ Keywords: preclinical, adoptive cell transfer, natural killer cells, allogeneic peripheral blood mononuclear cells \\ Received: July 27, 2015 Accepted: January 01, 2016 Published: January 18, 2016
}

\section{ABSTRACT}

\begin{abstract}
Treatment of ovarian cancer, a leading cause of gynecological malignancy, has good initial efficacy with surgery and platinum/taxane-based chemotherapy, but poor long-term survival in patients. Inferior long-term prognosis is attributed to intraperitoneal spreading, relapse and ineffective alternate therapies. Adoptive cell therapy is promising for tumor remission, although logistical concerns impede widespread implementation. In this study, healthy PBMCs were used to examine the immune response in a mouse model with human ovarian cancer, where natural killer (NK) cells were found to be the effector cells that elicited an antitumor response. Presence of tumor was found to stimulate NK cell expansion in mice treated intraperitoneally with PBMC+Interleukin-2 (IL-2), as compared to no expansion in non-tumor-bearing mice given the same treatment. PBMC+IL-2 treated mice exhibiting NK cell expansion had complete tumor remission. To validate NK cell mediated anti-tumor response, the intratumoral presence of NK cells and their cytotoxicity was confirmed by immunohistochemistry and granzyme activity of NK cells recovered from the tumor. Collectively, this study highlights the significance of NK cell-cytotoxic response to tumor, which may be attributed to interacting immune cell types in the PBMC population, as opposed to clinically used isolated NK cells showing lack of anti-tumor efficacy in ovarian cancer patients.
\end{abstract}

\section{INTRODUCTION}

Ovarian cancer is the second leading gynecological cancer [1]. Although current chemotherapy using carboplatin-paclitaxel is effective as a first-line therapy, $70 \%$ of patients with advanced disease succumb to tumor relapse within less than five years, despite initial response to chemotherapy [2]. Thus, there is an urgency to develop strategies for prevention of tumor relapse and for increasing overall patient survival. The immune system is protective against ovarian cancer and thus, adjuvant immunotherapy post-surgery and with chemotherapeutics could be effective for preventing relapse and extending survival [3, 4]. Studies show the link between cancer stem cells and ovarian tumor recurrence, and that treatment with chemotherapeutics selects resistant tumor populations causing increased risk of tumor relapse $[5,6]$. Such resistant tumor cell populations are susceptible to lymphocyte-mediated lysis, particularly by NK cells
[7]. Therefore, NK cell-based adjuvant immunotherapy could have significant impact on sustaining remission and improving overall treatment efficacy.

Immunotherapy has impacted treatment of multiple cancers. Monoclonal antibody-based approaches are beneficial for long-term effects, but a challenge is potential escape by tumors [8]. Adoptive cell therapy (ACT) with various immune cell types, T cells, dendritic cells (DCs) or natural killer (NK) cells in clinical trials hold promise. A landmark publication in 2003 showed that the infiltration of $\mathrm{T}$ cells into ovarian tumors improved survival [4]. Since tumor infiltrating immune cells influence the severity and overall disease outcome, attempts have been made to adoptively transfer tumor infiltrating lymphocytes (TILs) after lymphodepletion with successful results in metastatic melanoma patients $[9,10]$. DCs can activate the adaptive immune system for a robust response against infection or against 
transformed cells, and trials using DCs are ongoing (Clinicaltrials.gov identifier: NCT01875653). DCs function as antigen presenting cells for naïve CD4 and CD8 T cells [11]. To circumvent the need for $\mathrm{T}$ cell "education", chimeric antigen receptor (CAR)-T methods can genetically modify patient $\mathrm{T}$ cells to express a targeting receptor for a tumor specific antigen. In contrast to naïve CD4 and CD8 T cells that need to be "educated" or engineered ex vivo, innate NK cells are naturally cytotoxic towards malignant cells [12]. In vitro studies show that resting NK cells from healthy donors target isolated tumor cells from the peritoneal ascites of ovarian carcinoma patients [13]. In this respect, ACT using cytolytic NK cells for cancer treatment is more advantageous since NK cells do not require prior sensitization with an antigen and are not limited to targeting only tumors that have a specific marker as in CAR-T methods [14].

Clinical studies for ovarian and breast cancer using intravenously (IV) delivered NK cells enriched by CD3 depletion of PBMCs from haploidentical donors failed to show in vivo NK cell expansion perhaps due to suppression by host regulatory $\mathrm{T}$ (Treg) cells or myeloidderived suppressor cells [15]. Therefore, there is still an insufficient understanding about factors required for NK cell expansion and in vivo persistence for successful clinical outcome. A previous pre-clinical study showed that intraperitoneally (IP) delivered enriched NK cells could have anti-tumor response against ovarian cancer, and that NK cell cytotoxicity may be affected by the mode of delivery that could bypass hurdles of NK cell homing to the tumor location [16].

The quality of immune response to ovarian cancer has a significant impact on disease prognosis [17-19]. In the context of a complete immune system, innate NK cells can have direct cytotoxicity towards transformed cells as well as interact with DCs to induce IFN- $\gamma$ production which primes Th1 cells [20] and further enhances cytotoxic T cell responses [21]. NK cells are indispensable for effective DC-based immunotherapy, as loss of NK cells has shown to result in defective tumor immunity [22]. Such studies highlight the importance of NK cell interactions with both, innate and adaptive immune cell types, to affect adaptive immunity for effective anti-tumor response.

Here, we examine $\mathrm{NK}$ and $\mathrm{T}$ cells' response to tumor as part of an unbiased whole PBMC population as opposed to treating with selectively enriched NK or T cell populations. The study examines the kinetics of effector subtypes involved in the acute anti-tumor response of innate and adaptive components of PBMCs and identifies NK cells as the main effector cell of PBMCs' response, acting as a first line of anti-tumor defense. It also highlights the importance and points to the need for further studies to delineate other interacting immune cell types to strategically employ them as an adjuvant regimen for a safe and effective NK cell-based immunotherapeutic approach.

\section{RESULTS}

\section{Treatment with unselected healthy PBMCs clears human ovarian tumors engrafted in mice}

The interplay among multiple immune cell types in response to the presence of a tumor is complex and is still poorly understood. To address the therapeutic effectiveness of unselected immune cells from normal donor PBMCs in response to the presence of tumor, NSG mice that were IP inoculated with $1 \times 10^{6}$ SKOV3/GFP-Luc cells were monitored for engraftment. Mice that showed engraftment 7 days post inoculation were then treated with human 'PBMC+IL-2' (IL-2 dose: $1,000 \mathrm{U}$ thrice weekly) or remained 'untreated'. Another group of non-tumor bearing mice was injected with PBMC+IL-2 as a control. Treatment effectiveness was assessed by monitoring tumor size and overall health for 7 weeks after starting treatments. Serial imaging (Figure 1a) shows significant differences in tumor progression between the 'untreated' and the 'PBMC+IL-2 treated' groups. Untreated mice succumbed to disease in $\sim 3$ weeks, whereas tumor-engrafted mice treated with PBMC + IL-2 showed reducing tumor burden. Figure 1a shows reduction of tumor size in the treated group and total luminescence flux from the peritoneal tumor images acquired after PBMC injections (Figure 1b) also demonstrates the effect of treatment compared with no treatment ( $p=0.0003$, two-way ANOVA). A clear survival difference was observed in PBMC-treated compared to untreated mice $(p=0.001$, Log-rank Test) (Figure 1c). Untreated mice were euthanized upon health deterioration including abdominal bloating due to ascites and hunched posture. Overall, tumor-engrafted mice treated IP with whole PBMCs showed tumor regression within 3 weeks of PBMC treatment as compared to the untreated mice that succumbed to the disease within the same time frame.

\section{Expansion of NK cells in peripheral blood and peritoneal cavity of PBMC treated tumor-engrafted mice}

To determine if the cytolytic effector components in the anti-tumor response with the PBMC+IL-2 treatment is due to a single or synergistic lymphocyte populations in the allografted PBMCs, levels of immune cells were serially tracked in mouse peripheral blood for up to 7 weeks after PBMC injection. Delivering unselected healthy donor PBMCs along with low-dose IL-2, the expansion of NK cells was observed in the blood of only mice seeded with tumor. The Tumor+PBMC+IL2 group had day 7 counts of 650 to $1600 \mathrm{NK}$ cells/mL, and numbers of $\mathrm{NK}$ cells/mL increased significantly at the day 14 time point. The PBMC+IL2 group typically had counts $<100 \mathrm{NK}$ cells/mL and did not increase significantly. Also, mice that were injected only with PBMCs+IL-2 but without any tumor, showed 
no engraftment of NK cells (Figure 2). In a subsequent experiment, sets of mice treated with PBMC+IL-2 were collected on days 7, 14 and 21 post-treatment to examine the human lymphocyte population and their interaction with the tumor in the intraperitoneal cavity (Figure 3 ). NK cells/ $\mathrm{mL}$ counts in peritoneal wash were $\leq 2600 \mathrm{NK}$ cells $/ \mathrm{mL}$ at day 7 , after having been injected directly into the peritoneal cavity, and significantly increased at day 14. Similar to the previous experiment, treatment of tumor-seeded mice with PBMC and IL-2 delivered IP resulted in reduced tumor and complete clearance by 21 days after initiation of treatment. For each of the mice sacrificed on days 7, 14 and 21, cells were collected from the intraperitoneal cavity and were analyzed by flow cytometry. As seen previously in the analysis of peripheral blood, NK cell expansion occurred only in the presence of tumor, and despite IL-2 stimulation, mice without any tumor did not show NK cell engraftment, expansion or persistence. NK cell number peaked at day 14, when tumor signal intensity was also at its peak (Figure 1b) and then started to decline as the tumor regressed. Data from the blood analyzed during this experiment is shown in Supplemental Figure 1 with a similar trend as seen in the blood analysis of the survival experiment (Figure 2). These results show that the presence of tumor elicits specific expansion of NK cells from an unselected PBMC population, in the presence of low dose IL-2.

The result presented above suggest the anti-tumor activity of NK cells as part of a PBMC population. Followup experiments were conducted with either isolated NK cells or with NK cell depleted PBMCs. Supplemental Figure 6 shows a survival curve and the peripheral blood concentrations of total lymphocytes, NK cells, NKT cells and $\mathrm{T}$ cells from these follow-up experiments. The survival curve of the mice treated with isolated NK cells overlapped a.

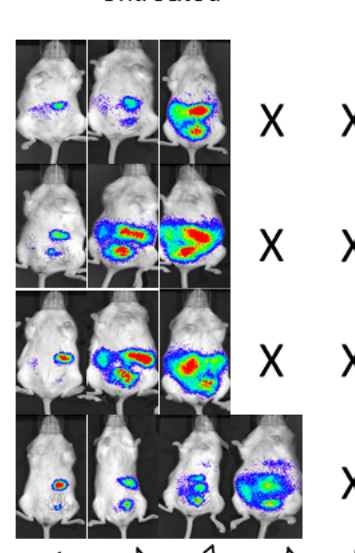

PBMC +IL-2 Treated
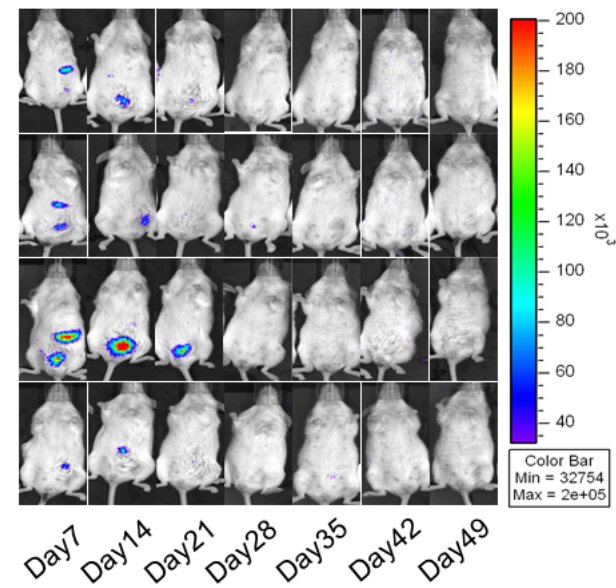

Days from PBMC injection

b.

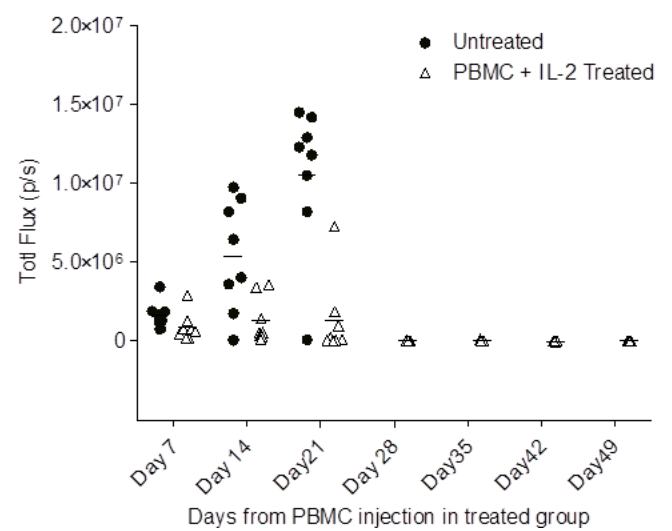

c.

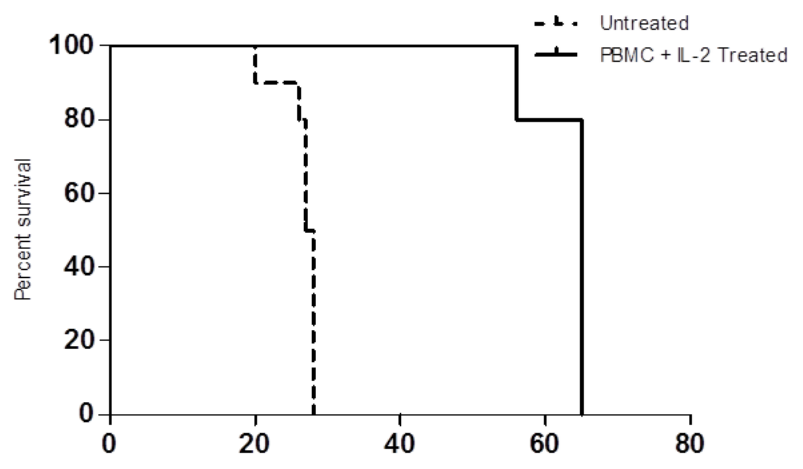

Days from tumor cell inoculation

Figure 1: SKOV-3/GFP-Luc tumor bearing mice show complete remission of tumor upon healthy human PBMC and IL-2 treatment. a. Bioluminescence of representative mice showing SKOV-3/GFP-Luc tumor burden over 7 weeks. Mice shown in the left panel were untreated, whereas the ones in the right panel were treated with PBMCs+IL-2 and monitored for tumor growth. b. Quantification of the tumor signal (total flux) obtained from imaging. P-value was $<0.001$ for the first three time-points (two-way ANOVA, GraphPad Prism). c. Curve showing the difference in overall survival of the untreated and PBMC treated mice from the day of tumor cell inoculation. $\mathrm{p}=0.001$ (Log-rank test, GraphPad Prism). 
a.

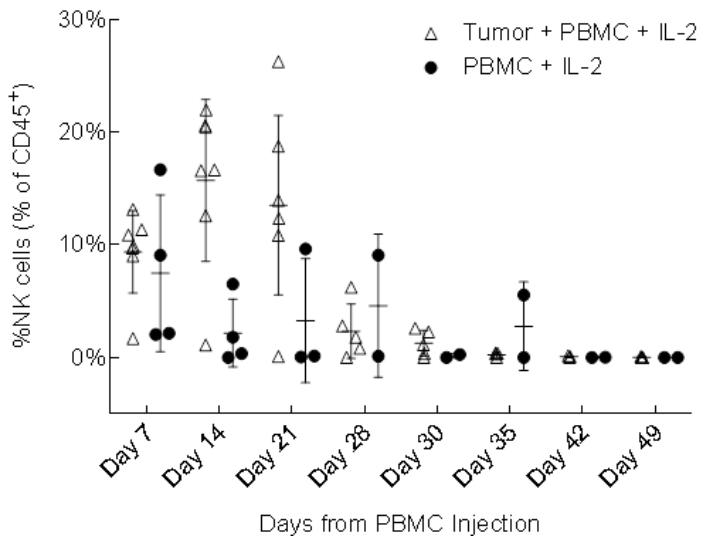

b.

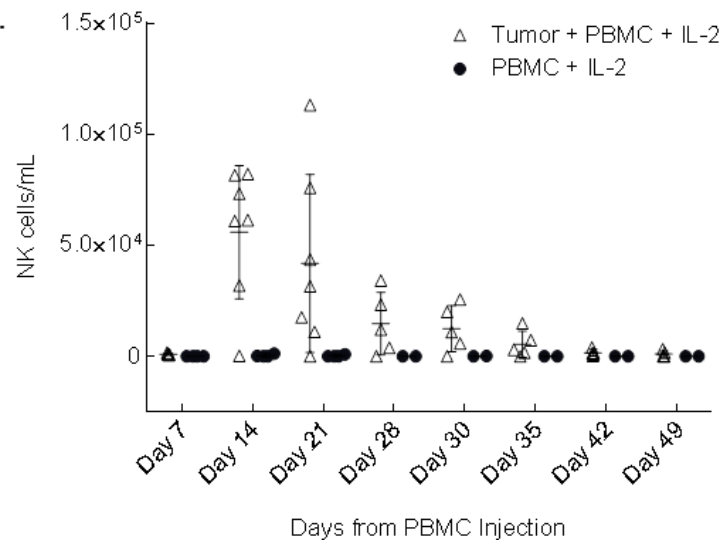

Figure 2: NK cells increase in peripheral blood in response to the presence of engrafted tumor. Plots show the fraction of human lymphocytes a. and concentration b. of human $\mathrm{CD}^{2} 5^{+}, \mathrm{CD} 56^{+}, \mathrm{CD} 3^{-} \mathrm{NK}$ cells in mouse peripheral blood at the indicated number of days after initiation of treatment. $\mathrm{p}=0.005$ (left); $\mathrm{p}=0.009$ (right) (two-way ANOVA, GraphPad Prism).

a.
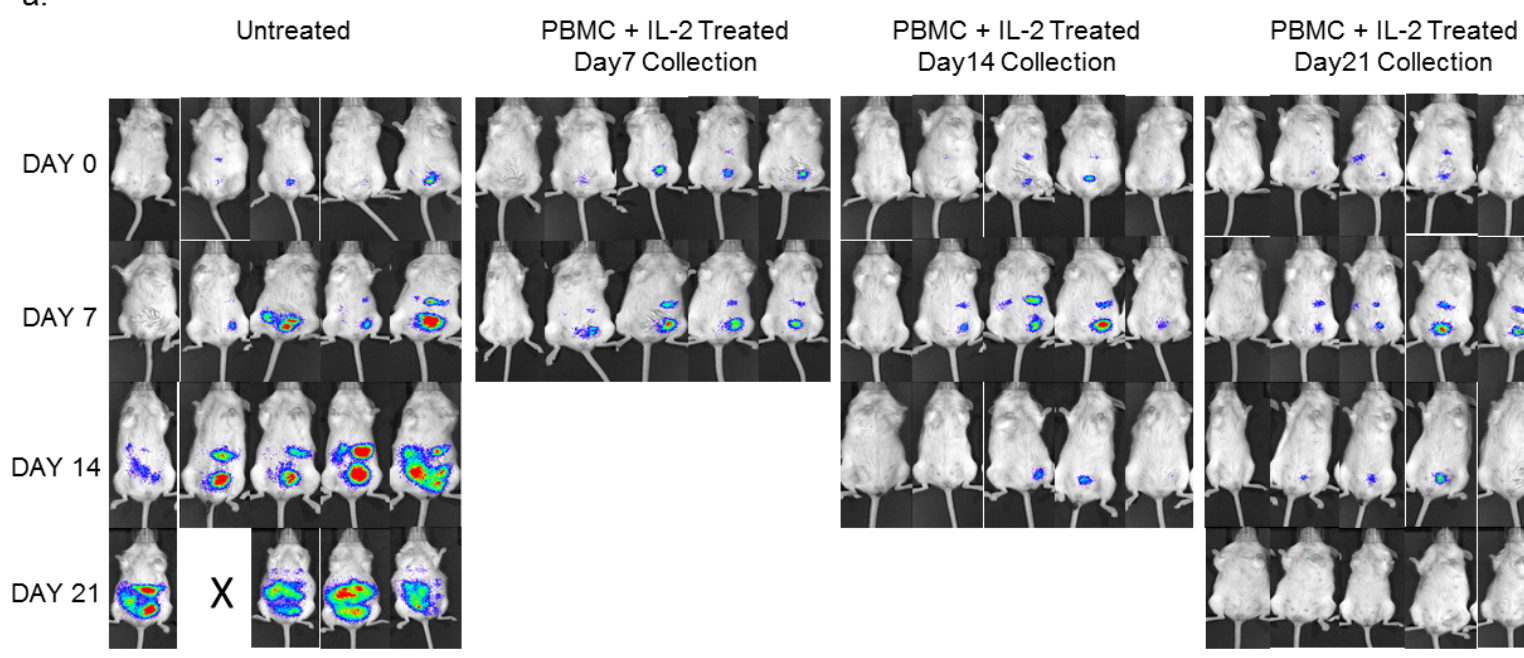

b.

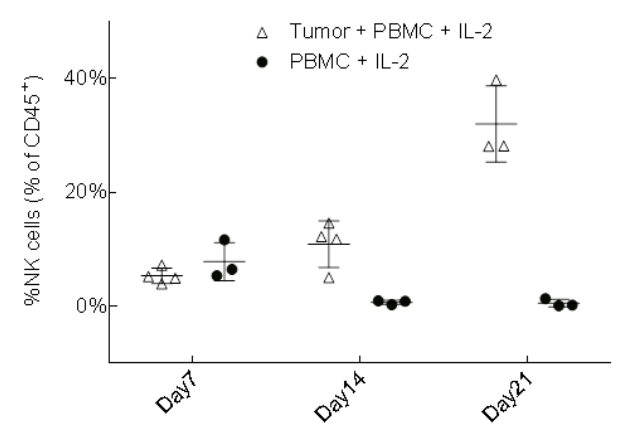

Days from PBMC Injection

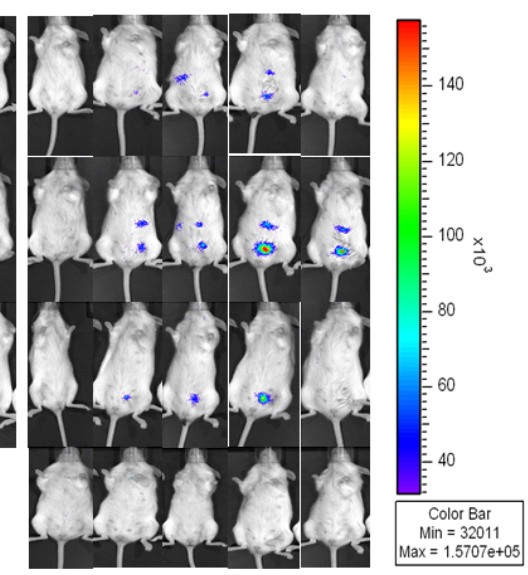

c.

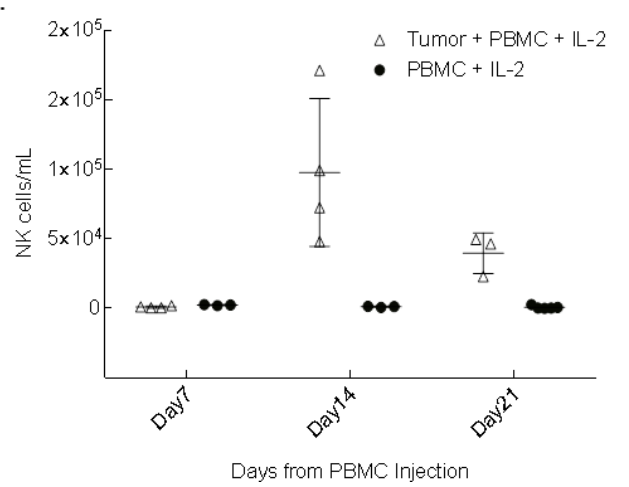

Figure 3: NK cells from whole PBMCs expand in the presence of tumor within the peritoneal cavity. a. After 10 days of SKOV-3/GFP-Luc engraftment, 3 groups were treated with $1 \times 10^{6}$ human PBMC+IL-2. Five mice were euthanized every week for 3 weeks for the analysis of peritoneal cavity wash. Another 3 groups of mice (not shown here) with no tumor and injected only with PBMCs + IL-2 were also collected along with the tumor bearing mice, as controls. b. Plots show the trend of expansion and reduction of NK cells in the peritoneal cavity expressed as fraction of human lymphocytes and c. as concentration over a time period of 3 weeks. $p=0.0001$ (b); $\mathrm{p}=0.0052$ (c) (two-way ANOVA, GraphPad Prism). 
with that of the untreated mice and we did not observe any immune cell growth in the blood of the mice treated with the isolated NK cells. This suggests that isolated NK cells did not have the necessary cross-talk with other cell populations in the PBMCs, such as the availability of IL-2 secreting T cells. The exogenous IL-2 provided was not sufficient to support NK cell expansion and thus, they did not thrive long enough to show any tumor cytotoxicity. In case of the NK cell depleted-PBMCs treated group, the survival curve overlapped with that of the whole PBMCs treated group. Although there were no significant NK cells/mL numbers, at day $14 \mathrm{NKT}$ cell expansion was seen in these mice (mean $\sim 1 \times 10^{5} \mathrm{NKT}$ cells $/ \mathrm{mL}$ ). In previous experiments, we have observed NKT cell expansion at day 14 (mean $\sim 1.8 \times 10^{4}$ NKT cells $/ \mathrm{mL}$ ), but higher numbers of NKT cells $/ \mathrm{mL}$ were observed in this experiment where NK cells were depleted.

\section{NK cells infiltrate tumor and show markers of cytotoxicity}

To corroborate that NK cell expansion in the presence of tumor in vivo and subsequent tumor remission was due to NK cell mediated cytotoxicity, shrinking tumors were collected from 14 day treated and untreated mice for analysis by histology. NKG2D was used as an NK cell marker because it is one of the main activating receptors on NK cells that recognizes various stress induced ligands on tumor cells [22]. Staining of a tumor nodule section (Day 14 after PBMC inoculation) from the PBMC+IL-2 treated group with anti-NKG2D showed positive NKG2D staining in the peripheral region of the nodule (Figure 4), which is indicative of intratumoral infiltration of NK cells. Granzyme release was observed
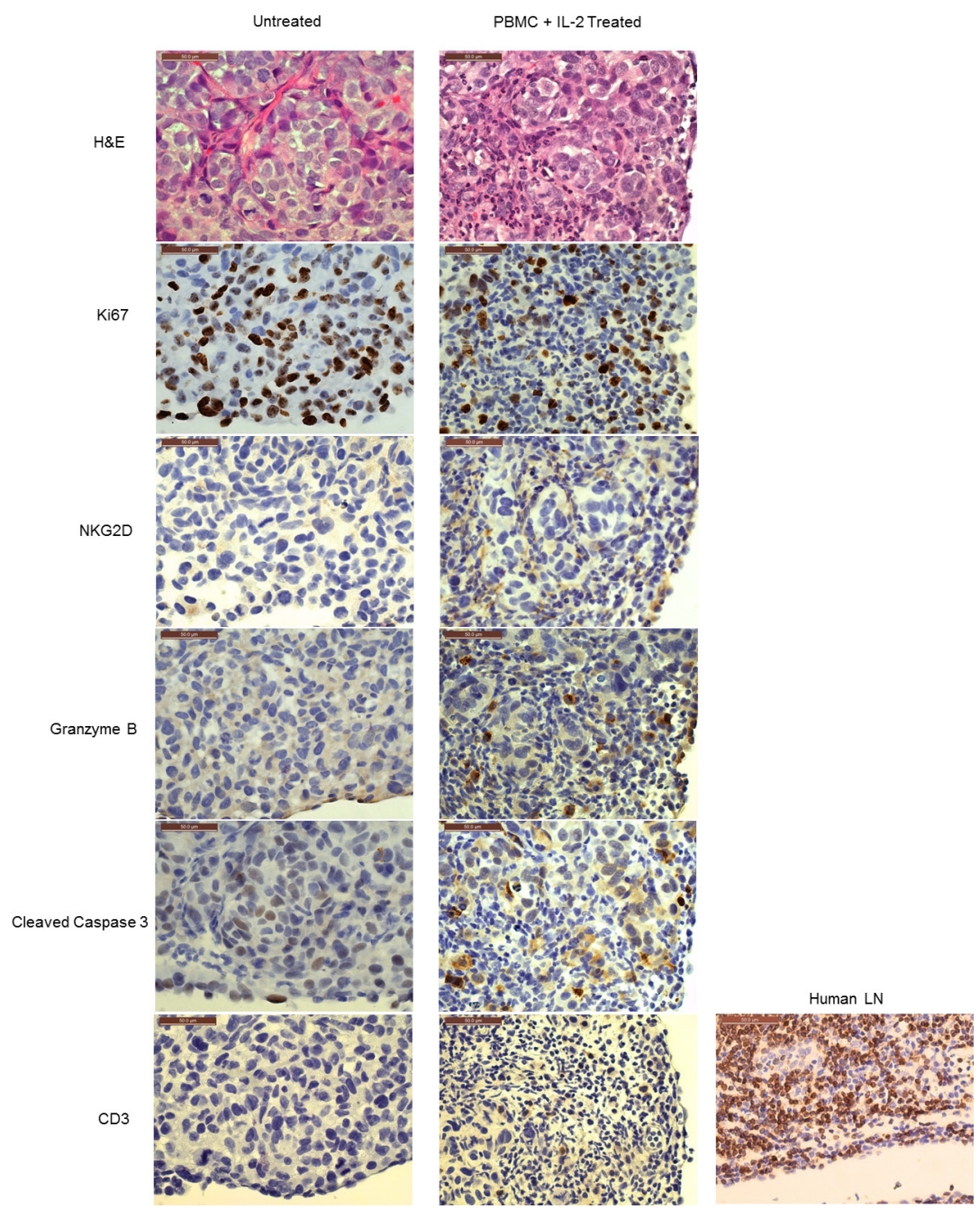

Figure 4: In vivo expanded NK cells infiltrate tumors and mediate tumor killing. The left panel shows representative images of a tumor nodule from the 'untreated' group of animals; the right panel shows that of 'PBMC+IL-2 treated' ones. The H\&E image in the top panel shows infiltrating immune cells compared to the untreated tumor. Tumor cells have Ki67 staining indicating proliferation. NKG2D staining shows NK cells within the tumor of the PBMC+IL-2 treated group. Granzyme B staining shows NK cell granules containing granzyme which mediate target cell lysis. Cleaved Caspase 3 staining indicates apoptosis in the tumor cells. The last panel shows the lack of CD3 staining in the same region of the tumor showing apoptosis. A human lymph node (LN) is shown as a positive control for the human CD3 antibody. All images were acquired using a 40X objective and scale bars represent a length of $50 \mu \mathrm{m}$. 
in the same region of the tumor. This is consistent with the presence of granzyme containing cytotoxic granules released from NK cells upon tumor cell recognition to mediate apoptosis by caspase dependent pathways [23]. Detection of cleaved caspase 3 by staining in the same region as that of granzyme release confirms NK cell induced apoptosis of tumor cells (Figure 4).

Since $\mathrm{T}$ cell expansion was observed in the peripheral blood (Supplemental Figure 2) and the peritoneal cavity (Supplemental Figure 3), their presence was also examined by staining with anti-CD3. CD3 staining was minimal (last panel in Figure 4) as compared to NKG2D staining in the same region of the tumor or as compared to CD3 staining in a human lymph node used as a positive control, thus confirming that the cytotoxicity markers were attributable to infiltrating NK cells killing tumors cells and not T cells. The NKG2D staining appears to be dim in the region shown, perhaps because of actively dying cells in that area due to granzyme release. A different region of the tumor shows clearer NKG2D staining (Supplemental Figure 4). These results indicate that $\mathrm{NK}$ cells expand in the vicinity of the tumors (peritoneal cavity) and infiltrate into the small tumor nodules to cause tumor regression observed in the PBMC + IL-2 treated mice.

\section{NK cells show direct cytotoxicity towards tumor cells ex vivo}

To confirm that the intratumoral NK cells are cytotoxic and not anergic, intratumoral perfusions were isolated from two tumor bearing mice being treated with PBMCs+IL-2 for 14 days and were tested for granzyme activity as discussed in the methods section. Flow cytometric analysis of granzyme activity in singlet vs. doublet tumor-lymphocyte conjugate populations was performed as previously described [24], to determine the cytotoxic response of intratumoral NK and T cells against fresh SKOV-3/GFP-Luc cells (Supplemental Figure 5). Differences in scatter properties (forward scatter height vs area plots) and CD45 intensity were used to determine the conjugates (tumor cells and immune cells) and singlet cell populations. Populations were further gated for $\mathrm{CD}^{+} 6^{+}$, $\mathrm{CD}^{-} \mathrm{NK}$ cells and $\mathrm{CD}^{+} \mathrm{T}$ cells, and for signal from granzyme activity. Figure 5 represents the events positive for granzyme activity seen in singlet and conjugate cells. Overall, significant difference ( $p=0.0002$, two-way ANOVA) was observed in the number of granzymepositive NK cells as compared to $\mathrm{T}$ cells with majority of the NK cells forming conjugates with tumor cells. These results show that isolated intratumoral NK cells are not anergic and highly cytotoxic towards SKOV-3/ GFP-Luc tumor cells, and gives strong evidence that the tumor shrinkage effect observed in vivo is due to cytolytic activity from NK cells and not $\mathrm{T}$ cells.

\section{DISCUSSION}

In this study, complete tumor remission and increased survival of tumor bearing NSG mice was observed when mice were treated with healthy donor derived PBMCs, as compared to the untreated ones. From the whole PBMC population injected, NK cell expansion was observed in the peritoneal cavity as well as in the peripheral blood of the tumor bearing mice, whereas no immune cell expansion or NK cell engraftment was seen in the control mice without any tumor. There was also a correlation between tumor shrinkage and decreasing

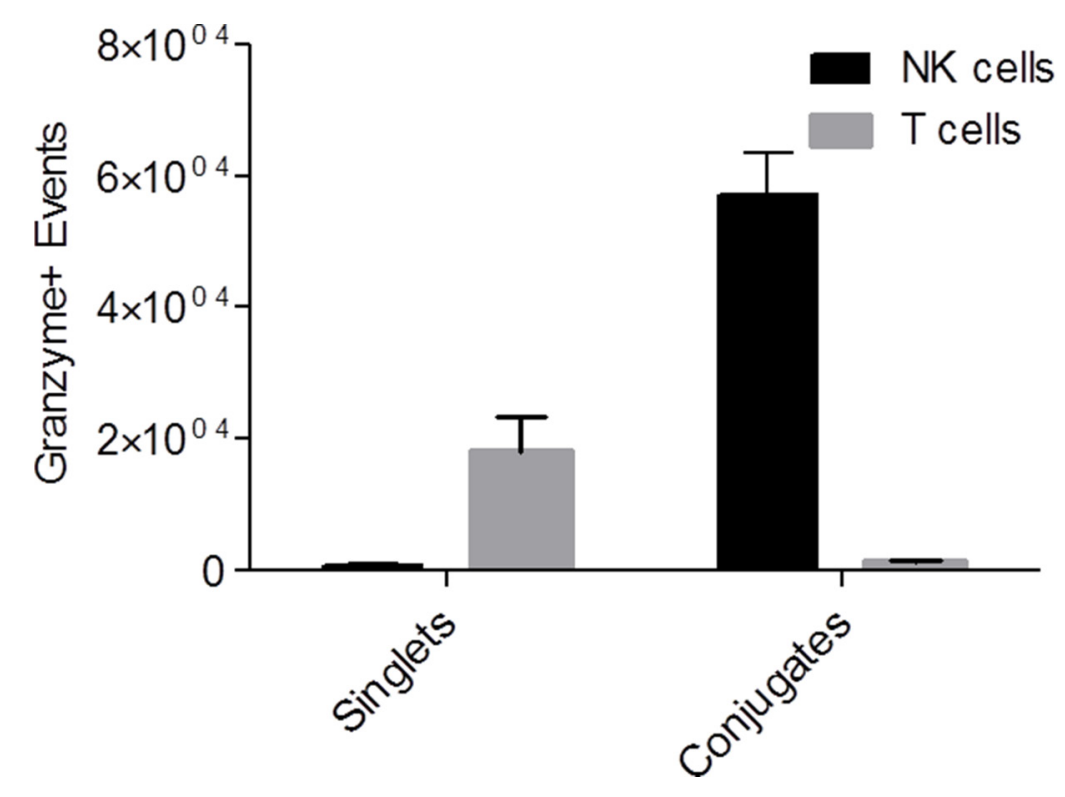

Figure 5: Granzyme assay showing actively cytotoxic NK cells perfused from tumors. Graph representing granzyme positive events of $\mathrm{NK}$ and $\mathrm{T}$ cells present in $\mathrm{CD}^{+} 5^{+}$singlet or conjugated populations upon perfusion from tumors. $\mathrm{p}=0.0002$ (two-way ANOVA). 
number of NK cells in the treated mice. Importantly, flow cytometry of granzyme activity of immune cells isolated from tumors showed that 1 ) there were more NK cells than $\mathrm{T}$ cells, 2) the majority of tumor conjugates were that of tumor-NK cells and 3 ) these tumor-NK cell conjugates had granzyme activity. These data collectively led us to conclude that NK cells were the effector cells responsible for the tumor regression and progression-free survival of the mice.

Previous studies with either allogeneic or autologous NK cell adoptive therapy have been inconsistent in producing successful clinical outcomes due to various reasons, most importantly the inability of NK cells to expand and persist in vivo [25]. A phase II study with IV delivered haploidentical NK cells in ovarian and breast cancer patients with recurrent disease following lymphodepleting chemotherapy showed sub-optimal NK cell expansion [15]. Another study with adoptively transferred autologous NK cells showed proliferation and persistence in vivo, but the NK cells failed to exhibit cytotoxicity due to immunosuppression without any overall benefit to the health of the patients [26]. A study in a xenograft mouse model showed progress with in vivo persistence of NK cells by changing the mode of delivery from IV to IP, but this study required very high starting dose of in vitro IL-2 pre-activated NK cells $\left(20 \times 10^{6}\right)$, enriched by $\mathrm{T}$ and $\mathrm{B}$ cell depletions of PBMCs, and high dose IL-2 (75,000 U/day IP daily for 3 weeks) in order to allow reduction in tumor burden with MA-148/GFP-Luc ovarian tumor cells [16]. In contrast, only $1 \times 10^{6}$ whole PBMCs (8-11\% NK cells) were injected IP along with very low dose of IL-2 (1,000 U thrice weekly) to induce complete SKOV-3/GFP-Luc tumor remission in this study. This suggests that a supportive or synergistic role of other cell types in the whole PBMC population is crucial for optimal NK cell cytotoxic activity against tumors. The followup experiment (Supplemental Figure 6) conducted with isolated NK cells or NK depleted PBMCs further supports the concept that immune cells work as a communal composition and the lack of a single cell type alters the stimulation of different immune cell type(s) as part of that composition and their potential anti-tumor response.

Cell types that are known to augment NK cell proliferation and maintenance are monocytes, DCs and $\mathrm{T}$ cells. Monocytes have been shown to aid in NK cell expansion mediated by some soluble factors [27]. Results from in vitro and clinical studies indicate that cytokines such as IL-2, 1L-12 and IL-21 enhance NK cell proliferation and effector functions such as IFN- $\gamma$ secretion $[28,29]$. DCs also play a role in NK cell effector functions like IFN- $\gamma$ production and cytolytic activity in vitro $[30,31]$. Mouse and human studies show that they mediate their support mainly through secretion of IL-12 $[32,33]$ and trans-presentation of membrane bound IL15 on mature DCs [30, 34-36]. In addition to triggering NK cell expansion and activation through a direct cell- to-cell contact, DCs can also influence NK cells by releasing exosomes $[37,38]$. A clinical study showed that vaccination with dendritic cell derived exosomes harboring NKG2D ligands and functional IL-15R $\alpha$, synergize for NK cell proliferation and activation in vitro and in patients [39]. In addition to monocytes and DCs, NK cells are also dependent on activated $\mathrm{CD}^{+} \mathrm{T}$ cells for the availability of IL-2, but secretion of IL-2 by these cells also causes Treg cell expansion as a negative feedback to prevent overstimulation of $\mathrm{CD}^{+} \mathrm{T}$ cells. Therefore, Tregs restrict the availability of IL-2 for NK cells causing suppression of NK cell expansion and activity [40,41], thus validating the effect of Treg depletion to enhance tumor suppression by NK cells $[42,43]$. In our study, the presence of T cells in the peritoneal cavity (Supplemental Figure 3) may have provided IL-2 for NK cell expansion in addition to lowdose exogenous IL-2. Apart from indirect inhibition of NK cells, Tregs can also directly suppress NK cells in a tumor microenvironment by secreting TGF- $\beta$, which is known to suppress NKG2D expression on NK cells and mitigate tumor cell lysis [44]. From these examples, it is clear that cross talk of NK cells with different components of the PBMCs plays a critical role in the regulation of NK cell proliferation and function.

In our study, NK cells infiltrated into small diffuse tumor nodules in the peritoneal cavity and were able to degranulate to induce cytotoxicity. This models residual tumor burden or diffuse persistent ovarian carcinoma where tumor sizes are small and perhaps not as immunosuppressive as compared to an advanced tumor. For the treatment of advanced tumors, NK cell therapy in combination with other treatment modalities may be required to achieve maximal clinical benefit. Various types of combination immunotherapies have been carried out in ovarian carcinoma patients, with promising results [45]. A new clinical trial is recruiting patients with recurrent ovarian and fallopian tube cancer along with other primary peritoneal carcinomas, for a phase 1 study looking at the effect of in vivo suppression of $\mathrm{T}$ cells by using an indoleamine 2,3-dioxygenase (IDO) inhibitor, along with IP delivery of haploidentical NK cells (Clinicaltrials.gov identifier: NCT02118285). IDO is prevalent in $\sim 56 \%$ of ovarian carcinomas and is associated with an immunosuppressive tumor microenvironment [46]. Also, in the ID8-VEGF ovarian carcinoma mouse model, decreased proliferation and the inability of the tumor infiltrating lymphocytes to produce cytokines was attributed to the expression of CTLA-4 and PD-1 makers [47]. Therefore, a combination of NK cell therapy with other strategies such as checkpoint inhibition may result in better clinical responses. For HLA expressing tumors that are resistant to NK cell lysis, combining NK cell therapy with an anti-KIR receptor antibody may be useful [48]. Because of the heterogeneous nature of tumors and a myriad of escape mechanisms used by tumors to evade the immune system, combining different approaches 
that inhibit mechanisms for immune escape in eclectic combinations is a rational approach.

NK cell function is regulated by a balance between various combinations of activating and inhibitory signals that allow the diversity of NK cell repertoire in an individual, but the link between NK cell diversity and NK cell function is only starting to be understood. In an analysis of peripheral blood NK cells, a study showed that there are 6-30,000 phenotypic variants of NK cells in an individual [49]. While inhibitory receptor expression is largely genetically governed, activation receptors are influenced by environmental factors in an individual [49]. The whole PBMC approach in our study rather than using enriched, in vitro IL-2 pre-activated NK cells, likely resulted in the conservation of the diversity of the activated NK cell repertoire due intercellular stimulation of NK cells with other components of the healthy donor PBMC population and tumor. This may have contributed to effectiveness of tumor induced NK cell expansion in vivo from low starting percentages $(8-11 \%)$ to $\sim 20 \% \mathrm{NK}$ cells in the total lymphocyte population, and stimulation of anti-tumor efficacy. In adoptive transfer of isolated, preactivated NK cells, the lack of intercellular stimulation from other lymphocyte types and potential skewing of NK cell population during in vitro pre-activation may possibly account for the lack of NK cell expansion and maximizing anti-tumor efficacy in ovarian carcinoma patients. For an effective anti-tumor response, all components are likely to have a role, but the absence of NK cells has shown to impair tumor rejection [50].

Results from these studies suggest that the cross talk of NK cells with other cell types in the PBMC population may be responsible for $\mathrm{NK}$ cell proliferation, infiltration into small and diffuse tumors and anti-tumor cytotoxicity. This may be an important consideration for utilizing NK cell-based immunotherapy as an adjuvant after surgical tumor resection and frontline chemotherapy, especially in the case of tumors where the elimination of residual tumor could be very significant to prevent relapse, such as in ovarian cancer. Therefore, this study provides a proof-of-principle for the therapeutic potential of NK cells in the anti-tumor response and highlights the importance of further investigating cell-to-cell interactions within PBMC population, based on which, different combination approaches could be designed to significantly attenuate tumor evasion.

\section{MATERIALS AND METHODS}

\section{Animals}

Animal care and use was at the Association for Assessment and Accreditation of Laboratory Animal Care International (AAALAC)-accredited University of Central Florida (UCF) vivarium. Protocols were approved by the UCF Institutional Animal Care and Use Committee (IACUC) and were compliant with NIH guidelines. NOD.
Cg-Prkdc $c^{\text {scid }} I l 2 r \gamma^{\text {tm } 1 W j l} / \mathrm{SzJ}$ (NSG) mice were obtained through an institutional material transfer agreement with Jackson Laboratory, Bar Harbor, Maine, USA.

\section{Human ovarian tumor cells and engraftment in mice}

Human ovarian tumor cell line SKOV-3/GFP-Luc was obtained from Cell BioLabs, Inc. (San Diego, CA, USA). The cell line was passaged in an NSG mouse once, then sorted for GFP expressing tumor cells using a cell sorter (FACSAria, BD Biosciences, Franklin Lakes, NJ, USA). NSG mice were IP injected with $1 \times 10^{6}$ SKOV-3GFP/Luc cells and tumors seeded for 7-10 days. Tumor growth was monitored by in vivo bioluminescent imaging.

\section{In vivo imaging}

SKOV-3/GFP-Luc tumor formation and growth was monitored using the Xenogen In vivo Imaging System (IVIS, Caliper Life Sciences, Hopkinton, MA, USA). Luciferin (Gold Biotechnology, Inc., St. Louis, MO, USA,) was IP injected at a dose of $0.15 \mathrm{mg} / \mathrm{g}$ body weight fifteen minutes prior to imaging. Maximum $/$ minimum of the luminescence signal intensity was adjusted to be the same throughout the experiment (Living Image software, Perkin Elmer, Waltham, MA, USA).

\section{Human PBMCs and in vivo treatments}

Human PBMCs were obtained from healthy human donor source leukocytes (OneBlood, Orlando, FL, USA) by standard Ficoll gradient. Isolated PBMCs were tested for viability using Annexin $\mathrm{V}$ staining and $\mathrm{NK}$ cell percentages by flow cytometry using a BD Accuri C6, BD Biosciences, San Jose, CA, USA) following staining by CD45, CD3 and CD56 antibodies. The initial NK cell percentages for PBMCs used in the experiments were $8-11 \%$ of total $\mathrm{CD} 45^{+}$cells. After tumor engraftment (7-10 days after inoculation), $1 \times 10^{6}$ viable PBMCs and 1,000 U human IL-2 (Preprotech, Rocky Hill, NJ, USA) were IP injected. 1,000 U IL-2 injections were given to mice weekly on Monday, Wednesday and Friday throughout the experiments. The 'untreated' mice in the experiments comprised of groups that were IP injected with 1) IL-2, 2) PBS or 3) were not treated with anything. All these mice behaved exactly the same with respect to the progression of the disease and their survival. Therefore, for the ease of representation, they are represented as one group and called as 'untreated' (with respect to PBMCs).

\section{Flow cytometry}

Fifteen minutes after subcutaneous saline injections to hydrate the animals, $<100 \mu \mathrm{L}$ of peripheral mouse blood was collected by submandibular bleeds once a week. 50 $\mu \mathrm{L}$ of blood was treated with red blood cell lysis buffer 
and stained with fluorophore conjugated antibodies against hCD45 (eBiosciences, San Diego, CA, USA), hCD56 (Miltenyi Biotec, Bergisch Gladbach, Germany) and hCD3 (Beckman Coulter, Brea, CA, USA). Peritoneal washes obtained by flushing $\sim 2 \mathrm{~mL}$ PBS in the peritoneal cavity were also processed similarly (i.e., $50 \mu \mathrm{L}$ of sample analyzed). Analysis of the data was done using the software Flowlogic (Inivai Technologies, Victoria, Australia). All NK cells (CD56 $\left.{ }^{+}, \mathrm{CD}^{-}\right)$were first gated on human $\mathrm{CD}_{4} 5^{+}$cells to find percent NKs, and based on the total number of events shown on the plots and the total volume of blood analyzed, the "cells/mL" numbers were obtained $(\mathrm{NK}$ cells $/ \mathrm{mL}=$ number of $\mathrm{NK}$ events/volume of blood analyzed).

\section{Histology}

Tumors were fixed in 10\% neutral-buffered formalin (Surgipath, Leica, Buffalo Grove, IL). Tissue was processed, embedded in paraffin blocks and $5 \mu \mathrm{m}$ sections were stained. Immunohistochemistry (IHC) was done using Leica's BondMax automated immunostainer. Antibodies included NKG2D (GeneTex, Inc., Irvine, CA, USA). Granzyme B (Spring Bioscience, Pleasanton, CA, USA), Cleaved Caspase 3 (Cell Signaling Technology, Inc., Danvers, MA, USA), CD3 (Abcam Cambridge, MA, USA). The images shown in Figure 4 are from sequential sections from the blocks and represent the same peripheral region of the tumor, three dimensionally.

\section{Granzyme assay}

From the tumors of mice treated for 14 days with PBMCs, cells were perfused using 18 gauge needles. They were washed and incubated with SKOV-3/GFP-Luc cells (50,000/well) on a 96-well plate for 1 hour. Cells were stained with anti-CD45, -CD3 and -CD56 antibodies in a granzyme substrate (PanToxiLux kit; Oncolmmunin, Gaithersburg, MD, USA). After $30 \mathrm{~min}$ incubation with antibodies, samples were analyzed using flow cytometry (CantoII, BD Biosciences) and Flowlogic software. To analyze the granzyme substrate signal (Fluorescein isothiocyanate, FITC) in the background fluorescence from the endogenous green fluorescent protein (GFP) of SKOV-3/ GFP-Luc cells present in the NK cell (or T cell)-SKOV-3/ GFP-Luc conjugates, a sample without granzyme substrate was used to define the fluorescent intensity for events correlating to cells that lack granzyme activity (Figure 4b).

\section{ACKNOWLEDGMENTS}

This work was supported by a Florida Department of Health Bankhead Coley Cancer Grant (4BB06) to A.J.C., a University of Central Florida (UCF) College of Medicine Competitive Research Grant to D.A.A., a donation from the Jean Bryant Jessie Memorial Ovarian Cancer Research Fund to D.A.A., and startup-funds from
UCF awarded to D.A.A. Thank you to Dr. Susan Blaydes Ingersoll (Florida Hospital Cancer Institute, Orlando, FL) for scientific input in the initial stages of the study. UCF Burnett School of Biomedical Sciences shared core equipment resources for animal care, histology, flow cytometry and confocal imaging were used for this study.

\section{CONFLICTS OF INTEREST}

The authors declare no conflicts of interest.

\section{REFERENCES}

1. Jemal A, Bray F, Center MM, Ferlay J, Ward E, Forman D. Global Cancer Statistics. CA: A Cancer Journal for Clinicians. 2011; 61:69-90.

2. Ushijima K. Treatment for recurrent ovarian cancer-at first relapse. Journal of Oncology. 2010; 2010:497429.

3. Braly P, Nicodemus CF, Chu C, Collins Y, Edwards R, Gordon A, McGuire W, Schoonmaker C, Whiteside T, Smith LM, Method M. The Immune adjuvant properties of front-line carboplatin-paclitaxel: a randomized phase 2 study of alternative schedules of intravenous oregovomab chemoimmunotherapy in advanced ovarian cancer. Journal of Immunotherapy. 2009; 32:54-65.

4. Zhang L, Conejo-Garcia JR, Katsaros D, Gimotty PA, Massobrio M, Regnani G, Makrigiannakis A, Gray H, Schlienger K, Liebman MN, Rubin SC, Coukos G. Intratumoral $\mathrm{T}$ cells, recurrence, and survival in epithelial ovarian cancer. The New England Journal of Medicine. 2003; 348:203-213.

5. Rizzo S, Hersey JM, Mellor P, Dai W, Santos-Silva A, Liber D, Luk L, Titley I, Carden CP, Box G, Hudson DL, Kaye SB, Brown R. Ovarian cancer stem cell-like side populations are enriched following chemotherapy and overexpress EZH2. Molecular Cancer Therapeutics. 2011; 10:325-335.

6. Zhan Q, Wang C, Ngai S. Ovarian cancer stem cells: a new target for cancer therapy. Biomed Res Int. 2013; 2013:916819.

7. Koh J, Lee SB, Park H, Lee HJ, Cho NH, Kim J. Susceptibility of CD24(+) ovarian cancer cells to anticancer drugs and natural killer cells. Biochemical and Biophysical Research Communications. 2012; 427:373-378.

8. Marincola FM, Jaffee EM, Hicklin DJ, Ferrone S. Escape of human solid tumors from T-cell recognition: molecular mechanisms and functional significance. Advances in Immunology. 2000; 74:181-273.

9. Dudley ME, Wunderlich JR, Robbins PF, Yang JC, Hwu P, Schwartzentruber DJ, Topalian SL, Sherry R, Restifo NP, Hubicki AM, Robinson MR, Raffeld M, Duray P, Seipp CA, Rogers-Freezer L, Morton KE, et al. Cancer regression and autoimmunity in patients after clonal repopulation with antitumor lymphocytes. Science. 2002; 298:850-854. 
10. Yee C, Thompson JA, Byrd D, Riddell SR, Roche P, Celis E, Greenberg PD. Adoptive T cell therapy using antigenspecific CD8 $+\mathrm{T}$ cell clones for the treatment of patients with metastatic melanoma: in vivo persistence, migration, and antitumor effect of transferred T cells. Proceedings of the National Academy of Sciences of the United States of America. 2002; 99:16168-16173.

11. Steinman RM. Decisions about dendritic cells: past, present, and future. Annu Rev Immunol. 2012; 30:1-22.

12. Srivastava S, Lundqvist A, Childs RW. Natural killer cell immunotherapy for cancer: a new hope. Cytotherapy. 2008; 10:775-783.

13. Carlsten M, Bjorkstrom NK, Norell H, Bryceson Y, van Hall T, Baumann BC, Hanson M, Schedvins K, Kiessling R, Ljunggren HG, Malmberg KJ. DNAX accessory molecule-1 mediated recognition of freshly isolated ovarian carcinoma by resting natural killer cells. Cancer Research. 2007; 67:1317-1325.

14. Childs RW, Berg M. Bringing natural killer cells to the clinic: ex vivo manipulation. Hematology / the Education Program of the American Society of Hematology American Society of Hematology Education Program. 2013; 2013:234-246.

15. Geller MA, Cooley S, Judson PL, Ghebre R, Carson LF, Argenta PA, Jonson AL, Panoskaltsis-Mortari A, Curtsinger J, McKenna D, Dusenbery K, Bliss R, Downs LS, Miller JS. A phase II study of allogeneic natural killer cell therapy to treat patients with recurrent ovarian and breast cancer. Cytotherapy. 2011; 13:98-107.

16. Geller MA, Knorr DA, Hermanson DA, Pribyl L, Bendzick L, McCullar V, Miller JS, Kaufman DS. Intraperitoneal delivery of human natural killer cells for treatment of ovarian cancer in a mouse xenograft model. Cytotherapy. 2013; 15:1297-1306.

17. Haskill S, Becker S, Fowler W, Walton L. Mononuclearcell infiltration in ovarian cancer. I. Inflammatory-cell infiltrates from tumour and ascites material. Br J Cancer. 1982; 45:728-736.

18. Sato E, Olson SH, Ahn J, Bundy B, Nishikawa H, Qian F, Jungbluth AA, Frosina D, Gnjatic S, Ambrosone C, Kepner J, Odunsi T, Ritter G, Lele S, Chen YT, Ohtani H, et al. Intraepithelial CD8+ tumor-infiltrating lymphocytes and a high $\mathrm{CD} 8+/$ regulatory $\mathrm{T}$ cell ratio are associated with favorable prognosis in ovarian cancer. Proceedings of the National Academy of Sciences of the United States of America. 2005; 102:18538-18543.

19. Leffers N, Gooden MJ, de Jong RA, Hoogeboom BN, ten Hoor KA, Hollema H, Boezen HM, van der Zee AG, Daemen T, Nijman HW. Prognostic significance of tumorinfiltrating T-lymphocytes in primary and metastatic lesions of advanced stage ovarian cancer. Cancer Immunol Immunother. 2009; 58:449-459.
20. Martin-Fontecha A, Thomsen LL, Brett S, Gerard C, Lipp M, Lanzavecchia A, Sallusto F. Induced recruitment of NK cells to lymph nodes provides IFN-gamma for T(H)1 priming. Nature Immunology. 2004; 5:1260-1265.

21. Kos FJ, Engleman EG. Requirement for natural killer cells in the induction of cytotoxic $\mathrm{T}$ cells. Journal of Immunology. 1995; 155:578-584.

22. Bouwer AL, Saunderson SC, Caldwell FJ, Damani TT, Pelham SJ, Dunn AC, Jack RW, Stoitzner P, McLellan AD. NK cells are required for dendritic cell-based immunotherapy at the time of tumor challenge. Journal of Immunology. 2014; 192:2514-2521.

23. Kagi D, Ledermann B, Burki K, Seiler P, Odermatt B, Olsen KJ, Podack ER, Zinkernagel RM, Hengartner H. Cytotoxicity mediated by $\mathrm{T}$ cells and natural killer cells is greatly impaired in perforin-deficient mice. Nature. 1994; 369:31-37.

24. Kim GG, Donnenberg VS, Donnenberg AD, Gooding W, Whiteside TL. A novel multiparametric flow cytometrybased cytotoxicity assay simultaneously immunophenotypes effector cells: comparisons to a $4 \mathrm{~h} 51 \mathrm{Cr}-$ release assay. J Immunol Methods. 2007; 325(1-2):51-66.

25. Cheng M, Chen Y, Xiao W, Sun R, Tian Z. NK cell-based immunotherapy for malignant diseases. Cell Mol Immunol. 2013; 10:230-252.

26. Parkhurst MR, Riley JP, Dudley ME, Rosenberg SA. Adoptive transfer of autologous natural killer cells leads to high levels of circulating natural killer cells but does not mediate tumor regression. Clinical Cancer Research. 2011; 17:6287-6297.

27. Miller JS, Oelkers S, Verfaillie C, McGlave P. Role of monocytes in the expansion of human activated natural killer cells. Blood. 1992; 80:2221-2229.

28. Roda JM, Joshi T, Butchar JP, McAlees JW, Lehman A, Tridandapani S, Carson WE, 3rd. The activation of natural killer cell effector functions by cetuximab-coated, epidermal growth factor receptor positive tumor cells is enhanced by cytokines. Clinical Cancer Research. 2007; 13:6419-6428.

29. Parihar R, Nadella P, Lewis A, Jensen R, De Hoff C, Dierksheide JE, VanBuskirk AM, Magro CM, Young DC, Shapiro CL, Carson WE, 3rd. A phase I study of interleukin 12 with trastuzumab in patients with human epidermal growth factor receptor-2-overexpressing malignancies: analysis of sustained interferon gamma production in a subset of patients. Clinical Cancer Research. 2004; 10:5027-5037.

30. Fernandez NC, Lozier A, Flament C, Ricciardi-Castagnoli P, Bellet D, Suter M, Perricaudet M, Tursz T, Maraskovsky E, Zitvogel L. Dendritic cells directly trigger NK cell functions: cross-talk relevant in innate anti-tumor immune responses in vivo. Nat Med. 1999; 5:405-411. 
31. Walzer T, Dalod M, Robbins SH, Zitvogel L, Vivier E. Natural-killer cells and dendritic cells: "l'union fait la force". Blood. 2005; 106:2252-2258.

32. Borg C, Jalil A, Laderach D, Maruyama K, Wakasugi H, Charrier S, Ryffel B, Cambi A, Figdor C, Vainchenker W, Galy A, Caignard A, Zitvogel L. NK cell activation by dendritic cells (DCs) requires the formation of a synapse leading to IL-12 polarization in DCs. Blood. 2004; 104:3267-3275.

33. Sarhan D, Palma M, Mao Y, Adamson L, Kiessling R, Mellstedt H, Osterborg A, Lundqvist A. Dendritic cell regulation of NK-cell responses involves lymphotoxin-alpha, IL-12, and TGF-beta. Eur J Immunol. 2015; 45:1783-1793.

34. Stonier SW, Schluns KS. Trans-presentation: a novel mechanism regulating IL-15 delivery and responses. Immunol Lett. 2010; 127:85-92.

35. Ferlazzo G, Pack M, Thomas D, Paludan C, Schmid D, Strowig T, Bougras G, Muller WA, Moretta L, Munz C. Distinct roles of IL-12 and IL-15 in human natural killer cell activation by dendritic cells from secondary lymphoid organs. Proceedings of the National Academy of Sciences of the United States of America. 2004; 101:16606-16611.

36. Koka R, Burkett P, Chien M, Chai S, Boone DL, Ma A. Cutting edge: murine dendritic cells require IL-15R alpha to prime NK cells. Journal of Immunology. 2004; 173:3594-3598.

37. Chaput N, Flament C, Viaud S, Taieb J, Roux S, Spatz A, Andre F, LePecq JB, Boussac M, Garin J, Amigorena S, Thery C, Zitvogel L. Dendritic cell derived-exosomes: biology and clinical implementations. J Leukoc Biol. 2006; 80:471-478.

38. Reiners KS, Dassler J, Coch C, Pogge von Strandmann E. Role of Exosomes Released by Dendritic Cells and/or by Tumor Targets: Regulation of NK Cell Plasticity. Frontiers in Immunology. 2014; 5:91.

39. Viaud S, Terme M, Flament C, Taieb J, Andre F, Novault S, Escudier B, Robert C, Caillat-Zucman S, Tursz T, Zitvogel L, Chaput N. Dendritic cell-derived exosomes promote natural killer cell activation and proliferation: a role for NKG2D ligands and IL-15Ralpha. PloS One. 2009; 4:e4942.

40. Trzonkowski P, Zaucha JM, Mysliwska J, Balon J, Szmit E, Halaburda K, Bieniaszewska M, Mlotkowska M, Hellmann A, Mysliwski A. Differences in kinetics of donor lymphoid cells in response to G-CSF administration may affect the incidence and severity of acute GvHD in respective HLAidentical sibling recipients. Med Oncol. 2004; 21:81-94.

41. Gasteiger G, Hemmers S, Firth MA, Le Floc'h A, Huse M, Sun JC, Rudensky AY. IL-2-dependent tuning of NK cell sensitivity for target cells is controlled by regulatory $\mathrm{T}$ cells. The Journal of Experimental Medicine. 2013; 210:1167-1178.

42. Shimizu J, Yamazaki S, Sakaguchi S. Induction of tumor immunity by removing CD25+CD4+ T cells: a common basis between tumor immunity and autoimmunity. Journal of Immunology. 1999; 163:5211-5218.

43. Smyth MJ, Teng MW, Swann J, Kyparissoudis K, Godfrey DI, Hayakawa Y. CD4+CD25+ T regulatory cells suppress NK cell-mediated immunotherapy of cancer. Journal of Immunology. 2006; 176:1582-1587.

44. Ghiringhelli F, Menard C, Terme M, Flament C, Taieb J, Chaput N, Puig PE, Novault S, Escudier B, Vivier E, Lecesne A, Robert C, Blay JY, Bernard J, Caillat-Zucman $\mathrm{S}$, Freitas A, et al. CD4+CD25+ regulatory T cells inhibit natural killer cell functions in a transforming growth factor-beta-dependent manner. The Journal of Experimental Medicine. 2005; 202:1075-1085.

45. Chester C, Dorigo O, Berek JS, Kohrt H. Immunotherapeutic approaches to ovarian cancer treatment. J Immunother Cancer. 2015; 3:7.

46. Inaba $\mathrm{T}$, Ino $\mathrm{K}$, Kajiyama $\mathrm{H}$, Yamamoto $\mathrm{E}$, Shibata $\mathrm{K}$, Nawa A, Nagasaka T, Akimoto H, Takikawa O, Kikkawa F. Role of the immunosuppressive enzyme indoleamine 2,3-dioxygenase in the progression of ovarian carcinoma. Gynecol Oncol. 2009; 115:185-192.

47. Duraiswamy J, Kaluza KM, Freeman GJ, Coukos G. Dual blockade of PD-1 and CTLA-4 combined with tumor vaccine effectively restores T-cell rejection function in tumors. Cancer Research. 2013; 73:3591-3603.

48. Romagne F, Andre P, Spee P, Zahn S, Anfossi N, Gauthier L, Capanni M, Ruggeri L, Benson DM, Jr., Blaser BW, Della Chiesa M, Moretta A, Vivier E, Caligiuri MA, Velardi A, Wagtmann N. Preclinical characterization of 1-7F9, a novel human anti-KIR receptor therapeutic antibody that augments natural killer-mediated killing of tumor cells. Blood. 2009; 114:2667-2677.

49. Horowitz A, Strauss-Albee DM, Leipold M, Kubo J, Nemat-Gorgani N, Dogan OC, Dekker CL, Mackey S, Maecker H, Swan GE, Davis MM, Norman PJ, Guethlein LA, Desai M, Parham P, Blish CA. Genetic and environmental determinants of human NK cell diversity revealed by mass cytometry. Science Translational Medicine. 2013; 5:208ra145.

50. Kim S, Iizuka K, Aguila HL, Weissman IL, Yokoyama WM. In vivo natural killer cell activities revealed by natural killer cell-deficient mice. Proceedings of the National Academy of Sciences of the United States of America. 2000; 97:2731-2736. 